\title{
SOME GEOMETRIC PROPERTIES OF SPACES ASSOCIATED WITH MULTIPLE STABLE INTEGRALS
}

\author{
JERZY SZULGA
}

(Communicated by Dale Alspach)

ABSTRACT. We investigate properties of vector lattices of multiply integrable functions with respect to a symmetric stable process.

\section{INTRODUCTION}

In this note we describe certain properties of spaces $\mathbb{S}_{\alpha}$ of multiparameter functions that are multiply integrable with respect to a symmetric $\alpha$-stable Lévy process $X_{\alpha}(t), 0<\alpha<2$. Such spaces are special tensor products of $L^{\alpha}$ spaces, and

$$
\bigcap_{\beta>\alpha} L^{\beta} \hookrightarrow \mathbb{S}_{\alpha} \hookrightarrow L^{\alpha}
$$

where “ $\hookrightarrow$ " denotes the continuous embedding, but a closed characterization is unknown at this time, for dimensions higher than two.

As is known, the distribution and the existence of the single stochastic integral $X_{\alpha} f=\int f d X_{\alpha}$ depends only on the integral $\int|f|^{\alpha}$. In [KaS] there was derived a recursive procedure for multiple integrability, but, except for $d=1$ or $d=2$, it did not provide any closed formula for the integrability. For the record, the double stable integrable $\iint f d X_{\alpha} d X_{\alpha}$ exists if and only if

$$
\iint|f(s, t)|^{\alpha}\left(1+\ln _{+} \frac{|f(s, t)|^{\alpha}}{\int|f(u, t)|^{\alpha} d u \int|f(s, v)|^{\alpha} d v}\right) d s d t<\infty
$$

(cf. [RW], and for its discrete counterpart, cf. [CRW]). The condition is clearly equivalent to the double integrability of $|f|^{\alpha}$ with respect to $X_{1}$. Hence, it is clear, at least in the case $d=1$ or $d=2$, that the spaces $\mathbb{S}_{\alpha}$ are obtainable from one another by a convexification (or concavification) procedure

$$
\mathbb{S}_{\alpha}=\left\{f: f^{\alpha} \in \mathbb{S}_{1}\right\} \text {. }
$$

In spite of the lack of concrete representation of integrable functions, the latter

Received by the editors May 10, 1992.

1991 Mathematics Subject Classification. Primary 46B20, 60H05; Secondary 46B30, 60E07.

Key words and phrases. Multiple stochastic integral, Banach lattice, $F$-lattice, stable distribution, $L^{p}$-space, $p$-convexity, $p$-concavity, Convexification, Rademacher type.

(C) 1994 American Mathematical Society $0002-9939 / 94 \$ 1.00+\$ .25$ per page 
relation, obtained in a simple way in this note, allows us to examine properties of Banach or -F-lattices $\mathbb{S}_{\alpha}$, like reflexivity, "closeness" to $L^{\alpha}$-spaces, Rademacher type, convexity type, etc.

\section{NotATION AND KNOWN FACTS}

For two quantities $A=A(\theta)$ and $B=B(\theta)$ depending on some parameter $\theta$, we will write $A \asymp B(A \preccurlyeq B$, respectively) if there is a constant $c>0$ such that $c^{-1} A \leq B \leq c A$ (respectively, $A \leq c B$ ), uniformly for $\theta$. It will be clear from the context whether or not the constant $c$ depends on additional parameters, like dimension or the stability index $\alpha$.

Sequences of length $d$ will be denoted by a boldface character, e.g., $\mathbf{u}=$ $\left(u_{1}, \ldots, u_{d}\right)$. The relation $\mathbf{i}=\left(i_{1}, \ldots, i_{d}\right) \leq n$ will mean $\max _{1 \leq k \leq d} i_{k} \leq n$. For an $x \in \mathbb{R}$ and $p \in \mathbb{R}$ we write $x^{p} \stackrel{\mathrm{df}}{=}|x|^{p} \operatorname{sgn}(x)$.

Throughout the paper, $U$ and $V$, with or without subscripts, will denote random variables uniformly distributed on $[0,1]$, while $\varepsilon$ will be a Rademacher random variable, i.e., one taking the values \pm 1 with probabilities $\frac{1}{2}$. Note that the random variable $\varepsilon U^{-1 / \alpha}$ belongs to the domain of normal attraction of the symmetric $\alpha$-stable law [F]. Let $\left[U_{i k}\right]_{k \in \mathbb{N}, i=1, \ldots, d}$ and $\left[V_{i k}\right]_{k \in \mathbb{N}, i=1, \ldots, d}$ be two independent random matrices whose entries consists of i.i.d. random variables. Let a matrix $\left[\varepsilon_{i k}\right]_{k \in \mathbb{N}, i=1, \ldots, d}$ consist of independent Rademacher random variables and be also independent of both matrices of uniform random variables.

We will use the operator notation, $X f=\int f d X$, for stochastic integrals. A symmetric $\alpha$-stable Lévy process on $[0,1]$ with values in $\mathbb{R}^{d}$ is denoted by $\mathbf{X}=\mathbf{X}_{\alpha}=\left(X_{\alpha}^{1}, \ldots, X_{\alpha}^{d}\right)$. Given a function $\mathbf{u}=\mathbf{u}(t)=\left(u_{1}(t), \ldots, u_{d}(t)\right) \epsilon$ $L^{\alpha}\left([0,1] ; \mathbb{R}^{d}\right)$, the distribution of the vector-valued coordinatewise integral $\mathbf{X u}=\left(X^{1} u_{1}, \ldots, X^{d} u_{d}\right)$ is given uniquely by a Lévy measure $\nu_{\alpha}(d \mathbf{x})$ or, separating the Levy measure into the radial and angular components, by the corresponding spectral measure $\sigma_{\alpha}(d \mathbf{x})$ on the $(d-1)$-dimensional sphere $S_{d}$,

$$
\begin{aligned}
E \exp \left\{i \sum_{k=1}^{d} X_{k} u_{k}\right\} & =\exp \left\{-\int_{0}^{1} \int_{\mathbb{R}^{d}}(1-\cos (\mathbf{x} \cdot \mathbf{u}(t))) \nu_{\alpha}(d \mathbf{x}) d t\right\} \\
& =\exp \left\{-\int_{0}^{1} \int_{S_{d}}|\mathbf{x} \cdot \mathbf{u}(t)|^{\alpha} \sigma_{\alpha}(d \mathbf{x}) d t\right\},
\end{aligned}
$$

where $\mathbf{x} \cdot \mathbf{u}$ denotes the inner product in $\mathbb{R}^{d}$.

Let $\mathscr{D}$ be a linear space of functions on $[0,1]^{d}$ which vanish on diagonal hyperplanes and are symmetric with respect to permutations of their arguments. Let $\mathbb{X}: \mathscr{D} \rightarrow L_{0}$ be a linear operator. Consider the conditions:

(1) $f\left(t_{1}, \ldots, t_{d}\right)=f_{1}\left(t_{1}\right) \cdots f_{d}\left(t_{d}\right) \in \mathscr{D}$, whenever $f_{i} \in L^{\alpha}$, and $\mathbb{X} f \stackrel{\mathrm{df}}{=}$ $X^{1} f_{1} \cdots X^{d} f_{d}$.

(2) If $\left\{f_{n}\right\} \subset \mathscr{D}, f_{n} \rightarrow f$ in measure, and $\sup _{n}\left|f_{n}\right| \leq g \in \mathscr{D}$, then $f \in \mathscr{D}$ and $\mathbb{X} f_{n} \stackrel{\mathrm{P}}{\rightarrow} \mathbb{X} f$.

The space of integrable functions $\mathbb{S}_{\alpha}$ is, by definition, a maximal space $\mathscr{D}$ with the above properties, while the operator $\mathbb{X}_{\alpha}=X_{\alpha}^{1} \cdots X_{\alpha}^{d}=\mathbb{X}: \mathbb{S}_{\alpha} \rightarrow L_{0}$ is termed the multiple stable integral [Kas]. This axiomatic definition is independent of a particular construction of a multiple stable integral. 
Basic properties of the multiple stochastic integral and the space $\mathbb{S}_{\alpha}$, are gathered below. Recall that by an F-space we mean a linear space with a topology induced by a complete invariant metric (cf., e.g., [Ru, pp. 8, 9]). Denote

$$
\|f\|_{\alpha, s}=\left(E\left|\mathbb{X}_{\alpha} f\right|^{s}\right)^{1 / s},
$$

where $s$ is any positive number less than $\alpha$.

Theorem A. The following properties hold:

(i) The functional $\|\cdot\|_{\alpha, s}\left(\|\cdot\|_{\alpha, s}^{s}\right.$ if $\left.s<1\right)$ is a norm (s-norm, if $s<1$ ) on $\mathbb{S}_{\alpha}$, turning $\mathbb{S}_{\alpha}$ into a Banach or $F$-space. Moreover, for a fixed $\alpha$, the norms are equivalent for all $s \in(0, \alpha)$.

(ii) Decoupling principle. If $\widehat{X}_{\alpha}^{1}, \ldots, \widehat{X}_{\alpha}^{d}$ are independent copies of the coordinate processes $X_{\alpha}^{1}, \ldots, X_{\alpha}^{d}$, then

$$
\left\|\mathbb{X}_{\alpha} f\right\|_{\alpha, s} \asymp\left\|\widehat{X}_{\alpha}^{1} \cdots \widehat{X}_{\alpha}^{d} f\right\|_{\alpha, s} .
$$

In particular, the class of integrable functions $\mathbb{S}_{\alpha}$ does not depend on the Lévy or spectral measures.

(iii) The finite dimensional distributions of stochastic processes with a parameter space $\mathbb{S}_{\alpha}$,

$$
S_{\alpha}^{n}(f)=n^{-d / \alpha} \sum_{i \leq n} \frac{f\left(V_{1 i_{1}}, \ldots, V_{d i_{d}}\right)}{U_{1 i_{1}}^{1 / \alpha} \cdots U_{d i_{d}}^{1 / \alpha}} \varepsilon_{1 i_{1}} \cdots \varepsilon_{d i_{d}},
$$

converge weakly to the distribution of a multiple stable integral $\mathbb{X}_{\alpha} f$ with independent coordinate processes.

(iv) $\mathbb{S}_{\alpha}$ is isomorphic to an $F$-space $\left\{f:\left(S_{\alpha}^{n}(f)\right)\right.$ is bounded in $\left.L^{0}\right\}$, equipped with a complete metric generated by the modular

$$
\tilde{\rho}_{\alpha, s}(f)=\sup _{n}\left\|S_{\alpha}^{n}(f)\right\|_{s}
$$

(v) The modular $\tilde{\rho}_{s}$ is equivalent to

$$
\begin{aligned}
\rho_{\alpha, s}(f) & =\sup _{n} n^{-d / \alpha}\left\|\left(\sum_{\mathbf{i}}\left|f\left(V_{1 i_{1}}, \ldots, V_{1 i d}\right)\left(U_{1 i_{1}} \cdots U_{d i_{d}}\right)^{-1 / \alpha}\right|^{2}\right)^{1 / 2}\right\|_{s} \\
& =\sup _{n} n^{-d / \alpha}\left\|\left(\left.\left.\sum_{\mathbf{i}}|| f\left(V_{1 i_{1}}, \ldots, V_{d i_{d}}\right)\right|^{\alpha}\left(U_{1 i_{1}} \cdots U_{d i_{d}}\right)^{-1}\right|^{2 / \alpha}\right)^{\alpha / 2}\right\|_{s / \alpha}^{1 / \alpha}
\end{aligned}
$$

(vi) Under the natural ordering, $\mathbb{S}_{\alpha}$ becomes a vector lattice. The norm (or $s$ norm) induced by the modular $\rho_{\alpha, s}$ is monotone. Hence, $|f| \leq|g| \Rightarrow\|f\|_{\alpha, s} \preccurlyeq$ $\|g\|_{\alpha, s}$.

Proof. (i) [S1, Theorem 3.5; KS1, Theorem 2.5 and Corollary 2.3].

(ii) [KS3, Proposition 5.1].

(iii) [S3, Proposition 3.1 and Corollary 3.2].

(iv) The connection between modulars and metrics is described, for example, in [M, pp. 2, 3]. The statement was proved in [S2, Theorem 6.6] in the case of an $L^{0}$-metric. Here, we can switch to a more convenient $L_{s}$-norm, for any $0<s<\alpha$, due to hypercontractivity of the random variable $Z^{(\alpha)}=\varepsilon U^{-1 / \alpha}$ (cf. [S1, Theorem 3.1; KS1, Theorem 2.5 and Corollary 2.3]). 
(v) It suffices to apply the $d$-dimensional Khinchin inequality (cf., e.g., [KS2, inequality (2.4)].

(vi) Obviously.

Lemma 1. Let $0<q<p<r<\infty$. Then, uniformly in all real sequences $\left(a_{i}\right)$,

$$
\mathrm{E}\left(\sum_{i}\left(\left|a_{i}\right| U_{i}^{-1 / p}\right)^{r}\right)^{q / r} \asymp\left(\sum_{i}\left|a_{i}\right|^{p}\right)^{q / p} \asymp \mathrm{E} \sup _{i}\left(\left|a_{i}\right| U_{i}^{-1 / p}\right)^{q} .
$$

Proof. Let $1<p^{\prime}<\infty$, and let $\left(Y_{i}\right)$ be a sequence of independent random variables such that $\mathrm{P}(|Y|>t)=t^{-1 / p^{\prime}}$ for every $i$ and every $t \geq 1$. Then for every $r^{\prime}>p^{\prime}$, by [LT, Lemma 1.f.8], we have

$$
\mathrm{E}\left(\sum_{i}\left|a_{i}^{\prime} Y_{i}\right|^{r^{\prime}}\right)^{1 / r^{\prime}} \asymp\left(\sum_{i}\left|a_{i}^{\prime}\right|^{p^{\prime}}\right)^{1 / p^{\prime}} \asymp \mathrm{E} \sup _{i}\left|a_{i}^{\prime} Y_{i}\right|
$$

The lemma follows by substituting

$$
r^{\prime}=r / q, \quad p^{\prime}=p / q, \quad a_{i}^{\prime}=\left|a_{i}\right|^{q}, \quad Y_{i}=U_{i}^{-1 / p^{\prime}}=U^{-q / p} .
$$

Lemma 2. Let $r>p>q>0$, and let $\mathbf{U}=\left[U_{i k}\right]_{i=1, \ldots, d, k \in \mathbb{N}}$ be a random $m a$ trix of mutually independent random variables uniformly distributed on $[0,1]$.

(i) Let $\left\{A(\mathbf{i}): \mathbf{i} \in \mathbb{N}^{d}\right\}$ be a family of random variables independent of $\mathbf{U}$. Then, for every $q \in(0, p)$.

$$
\left\|\left(\sum_{\mathbf{i}}\left(|A(\mathbf{i})|\left(U_{1 i_{1}} \cdots U_{d i_{d}}\right)^{-1 / p}\right)^{r}\right)^{1 / r}\right\|_{q} \asymp\left\|\sup _{\mathbf{i}}|A(\mathbf{i})|\left(U_{1 i_{1}} \cdots U_{d i_{d}}\right)^{-1 / p}\right\|_{q} .
$$

(ii) Let $0<q<p<\infty$. Then

$$
\left\|\sum_{\mathbf{i}} \delta(\mathbf{i}) a(\mathbf{i})\left(U_{1 i_{1}} \cdots U_{d i_{d}}\right)^{-1 / p}\right\|_{q} \asymp\left\|\sum_{\mathbf{i}} a(\mathbf{i})\left(U_{1 i_{1}} \cdots U_{d i_{d}}\right)^{-1 / p}\right\|_{q},
$$

for all functions $a: \mathbb{N}^{d} \rightarrow \mathbb{R}_{+}, \delta: \mathbb{N}^{d} \rightarrow[-1,1]$.

Proof. (i) The relation " $\succcurlyeq "$ is obvious. To prove the relation " $\preccurlyeq$ ", we use Fubini's theorem which, in particular, allows us to consider nonrandom matrices $[A(i)]$. For simplicity, we prove the statement in the case $d=2$. The general case follows in the similar way. Denote by $E_{1}$ and $E_{2}$ the expectations corresponding (in terms of Fubini's theorem and product measures or, equivalently in this context, by means of conditioning) to independent random sequences $\left(U_{1 i}\right)$ and $\left(U_{2 j}\right)$, respectively. Put

$$
b_{j}=\left(\sum_{i}\left(|A(i, j)| U_{1 i}^{-1 / p}\right)^{r}\right)^{1 / r} \quad \text { and } \quad c_{i}=\sup _{j}|A(i, j)| U_{2 j}^{-1 / p}
$$


We have

$$
\begin{gathered}
\mathrm{E}\left(\sum_{i} \sum_{j}\left(|A(i, j)| U_{1 i}^{-1 / p} U_{2 j}^{-1 / p}\right)^{r}\right)^{q / r}=\mathrm{E}_{1} \mathrm{E}_{2}\left(\sum_{j}\left(b_{j} U_{2 j}^{-1 / p}\right)^{r}\right)^{q / r} \\
\preccurlyeq \mathrm{E}_{1} \mathrm{E}_{2}\left(\sup _{j}\left(b_{j} U_{2 j}^{-1 / p}\right)^{r}\right)^{q / r} \preccurlyeq \mathrm{E}_{2} \mathrm{E}_{1}\left(\sum_{i}\left(c_{i} U_{1 i}^{-1 / p}\right)^{r}\right)^{q / r} \\
\preccurlyeq\left\|\sup _{i j}|A(i, j)| U_{1 i}^{-1 / p} U_{2 j}^{-1 / p}\right\|^{q} .
\end{gathered}
$$

(ii) The statement follows from the generalized Khinchin inequality and statement (i) of this lemma.

\section{MAIN Results}

Theorem 1. Let $0<\alpha, \beta<2$ and $f:[0,1]^{d} \rightarrow \mathbb{R}$ be a symmetric function vanishing on diagonal hyperplanes.

(i) $f$ is $\mathbb{X}_{\alpha}$-integrable if and only if $|f|$ is $\mathbb{X}_{\alpha}$-integrable.

(ii) $f$ is $\mathbb{X}_{\alpha}$-integrable if and only if $f^{\alpha}$ is $\mathbb{X}_{1}$-integrable if and only if $f^{\alpha / \beta}$ is $\mathbb{X}_{\beta}$-integrable. Additionally, for any $s, 0<s<\alpha$,

$$
\mathrm{E}\left|\mathbb{X}_{\alpha} f\right|^{s} \asymp \mathrm{E}\left|\mathbb{X}_{1} f^{\alpha}\right|^{s / \alpha} \asymp \mathrm{E}\left|\mathbb{X}_{\beta} f^{\alpha / \beta}\right|^{s \beta / \alpha}
$$

Proof. By the decoupling principle, we may deal with stable processes with independent coordinates. By Theorem A(iii), (iv), it suffices to consider the random operators $S_{\alpha}^{n}(f)$ instead of the integrals $\mathbb{X}_{\alpha} f$. Thus Lemma 2(ii) yields statement (i).

(ii) We infer from Lemma 2(i) that the modulars

$$
\Lambda_{p, q, r}(A)=\left\|\left(\sum_{\mathbf{i}}\left|A(\mathbf{i})\left(U_{1 i_{1}} \cdots U_{d i_{d}}\right)^{-1 / p}\right|^{r}\right)^{1 / r}\right\|_{q},
$$

where $A: \mathbb{N}^{d} \rightarrow L_{0}$ and $r>p>q>0$, are equivalent for all $r$. By hypercontractivity of $U^{-1 / p}$ (cf. [S1, Theorem 3.1]), the modulars are also equivalent for all permissible $q$. Then, the substitutions $p=1, r=2 / \alpha, A=f^{\alpha}$ prove the former equivalence in (3). The latter equivalence in (3) follows a fortiori.

Theorem 1 enables us to reduce the investigation of the totality of spaces $\mathbb{S}_{\alpha}$ to that of a space marked by an arbitrarily selected index $\alpha$. We will give a precise meaning of this statement.

Let $p>0$. We call the norm $\|\cdot\| p$-convex if

$$
\left\|\left(\sum_{i}\left|f_{i}\right|^{p}\right)^{1 / p}\right\| \preccurlyeq\left(\sum_{i}\left\|f_{i}\right\|^{p}\right)^{1 / p}, \quad\left\{f_{i}\right\} \subset \mathbb{S}_{\alpha},
$$

and $p$-concave if the relation " $\preccurlyeq "$ in the above definition is replaced by the relation " $\geqslant "$. Observe that the function $\left(\sum_{i}\left|f_{i}\right|^{p}\right)^{1 / p}$ is well defined in $\mathbb{S}_{\alpha}$, since $\mathbb{S}_{\alpha}$ is a solid vector lattice (i.e., $|f| \leq g \in \mathbb{S}_{\alpha} \Rightarrow f \in \mathbb{S}_{\alpha}$ ). These notions 
are well known in the context of Banach lattices and indices $p \geq 1$ (cf. [Kri] or [LT, §1.d]). For an arbitrary F-lattice $\mathbb{L}$ of functions, define

$$
\mathbb{L}^{(p)}=\left\{f: f^{P} \in \mathbb{L}\right\} .
$$

If $\|\cdot\|$ denotes a homogeneous positive functional on $\mathbb{L}$, we also put

$$
\|f\|_{(p)}=\left\|f^{p}\right\|^{1 / p} .
$$

It is easy to check that, if $\|\cdot\|$ is $s$-convex and $r$-concave, then $\|\cdot\|_{(p)}$ is $p s$-convex and $p r$-concave. The operation $\mathbb{L} \mapsto \mathbb{L}^{(p)}$ is usually called the $p$ convexification if $p>1$, and $1 / p$-concavification if $p<1$ [LT, pp. 53-54].

Recall that a Banach space $\mathbb{L}$ is of Rademacher type $r$ if

$$
\mathrm{E}\left\|\sum_{i} x_{i} \varepsilon_{i}\right\| \preccurlyeq\left(\sum_{i}\left\|x_{i}\right\|^{r}\right)^{1 / r}, \quad\left\{x_{i}\right\} \subset \mathbb{L} .
$$

By a suitable concavification, it is easy to formulate an analogous property in the context of spaces $\mathbb{S}_{\beta}$ and $\beta \leq 1$.

In the one-dimensional situation, the spaces $\mathbb{S}_{1}$ and $L_{1}$ are identical. For a higher dimension, the structure of the space is no longer explicit, even though $\mathbb{S}_{1}$, for $d=2$, is clearly characterized by (1).

Theorem 2. Let $0<\alpha<2$.

(i) For $0<\beta<2,\left(\mathbb{S}_{\beta}\right)^{(\alpha / \beta)}=\mathbb{S}_{\alpha}$.

(ii) Except for $d=1$, there is no equivalent Banach lattice renorming of $\mathbb{S}_{1}$.

(iii) $\mathbb{S}_{\alpha}$ is $r$-concave for every $r \geq \alpha$ and $r$-convex for every $0<r<\alpha$. In particular, (a) multiple 1-stable integrals satisfy the relation

$$
\left(\mathrm{E}\left(\sum Y_{i}\right)^{s}\right)^{1 / s} \geq \sum\left(\mathrm{E} Y_{i}^{s}\right)^{1 / s} ;
$$

and (b) $\mathbb{S}_{\alpha}$ is $r$-uniformly smoothable for $1<r<\alpha$ and, thus, it is a reflexive (even superreflexive) Banach lattice.

(iv) $\mathbb{S}_{\alpha}$ is not of Rademacher type $\alpha$ although it is of Rademacher type $r$ for $1<r<\alpha$.

Proof. (i) follows immediately from Theorem 1(ii).

(ii) Let $\alpha>1$. Assume the contrary. If there were an equivalent Banach lattice norm on $\mathbb{S}_{1}$, it would be 1-convex. It suffices to construct a twodimensional counterexample based on the characterization (1).

Let $0<c_{i}<1$, and put $f_{i}=\dot{c}_{i} \mathbb{1}_{[i, i+1) \times[i-1, i)}$. Then, by (1), $\mathbb{X}_{1} \sum f_{i}$ converges in $L_{q}$ for some $q<1$ if and only if $\sum c_{i} \ln \left(1 / c_{i}\right)$ converges. On the other hand, $\sum i \mathbb{X}_{1} f_{i} \|_{q}$ converges if and only if $\sum c_{i}$ converges. It is easy to find a suitable sequence $\left(c_{i}\right)$, for instance, taking $c_{i}=\left(i \ln ^{1+\varepsilon} i\right)^{-1}, 0<\varepsilon<1$.

(iii) Let $\mathbb{X}_{\alpha}^{(d)}$ and $\mathbb{X}_{\alpha}^{(d-1)}$ be $d$ - and $(d-1)$-dimensional multiple stable integrals, respectively. Then the first of the following relations is another byproduct of the decoupling principle (Proposition 2), and the last one uses s-convexity 
of $L_{\alpha}$ for $s<\alpha$ :

$$
\begin{aligned}
\left\|\mathbb{X}_{\alpha}^{(d)} f\right\|_{s} & \asymp\left(E\left(\int_{0}^{1}\left|\mathbb{X}_{\alpha}^{(d-1)} f(\cdot, t)\right|^{\alpha} d t\right)^{s / \alpha}\right)^{1 / s} \\
& =\left\|\left(\int_{0}^{1}\left|\mathbb{X}_{\alpha}^{(d-1)} f(\cdot, t)\right|^{\alpha} d t\right)^{1 / \alpha}\right\|_{s} \geq\left(\int_{0}^{1}\left\|\mathbb{X}_{\alpha}^{(d-1)} f(\cdot, t)\right\|_{s}^{\alpha} d t\right)^{1 / \alpha}
\end{aligned}
$$

To prove the $r$-concavity of $\mathbb{S}_{\alpha}, r \geq \alpha$, we use the induction with respect to $d$ :

$$
\begin{aligned}
\left\|\mathbb{X}^{(d)}\left(\sum_{i}\left|f_{i}\right|^{r}\right)^{1 / r}\right\|_{s} & \succcurlyeq\left(\int_{0}^{1}\left\|\mathbb{X}_{\alpha}^{(d-1)}\left(\sum_{i}\left|f_{i}(\cdot, t)\right|^{r}\right)^{1 / r}\right\|_{s}^{\alpha} d t\right)^{1 / \alpha} \\
& \succcurlyeq\left(\int_{0}^{1}\left(\sum_{i}\left\|\mathbb{X}_{\alpha}^{(d-1)}\left|f_{i}(\cdot, t)\right|\right\|_{s}^{r}\right)^{\alpha / r} d t\right)^{1 / \alpha} \\
& \succcurlyeq\left(\sum_{i}\left(\int_{0}^{1}\left\|\mathbb{X}_{\alpha}^{(d-1)}\left|f_{i}(\cdot, t)\right|\right\|_{s}^{\alpha}\right)^{r / \alpha} d t\right)^{1 / r} \\
& \asymp\left(\sum_{i}\left\|\mathbb{X}^{(d)} f_{i}\right\|^{r}\right)^{1 / r},
\end{aligned}
$$

where, besides the inductive assumption, we have also used the $r$-concavity of $L_{\alpha}$.

To prove the convexity, we proceed as follows. Let $r<q<\alpha$. By Theorem 1 and the triangle inequality in $L_{q / r}$, we have

$$
\begin{aligned}
\left\|\left(\sum_{i}\left|f_{i}\right|^{r}\right)^{1 / r}\right\|_{\alpha, q} & =\left\|\mathbb{X}_{\alpha}\left(\sum_{i}\left|f_{i}\right|^{r}\right)^{1 / r}\right\|_{q} \asymp\left\|\mathbb{X}_{\alpha / r} \sum_{i}\left|f_{i}\right|^{r}\right\|_{q / r}^{1 / r} \\
& =\left\|\sum_{i} \mathbb{X}_{\alpha / r}\left|f_{i}\right|^{r}\right\|_{q / r}^{1 / r} \leq\left(\sum_{i}\left\|\mathbb{X}_{\alpha / r}\left|f_{i}\right|^{r}\right\|_{q / r}\right)^{1 / r} \\
& \asymp\left(\sum_{i}\left\|\mathbb{X}_{\alpha} f_{i}\right\|_{q}^{r}\right)^{1 / r}=\left(\sum_{i}\left\|f_{i}\right\|_{\alpha, q}^{r}\right)^{1 / r} .
\end{aligned}
$$

(iii)(a) Inequality (4) holds for $Y_{i}=\mathbb{X}_{1} g_{i}$ since $\mathbb{S}_{1}$ is 1 -concave. Note that this inequality shows that 1 -stable processes behave, in a sense, like nonnegative random processes, even though there is no positive 1-stable process.

(iii)(b) Cf. [LT, p. 101].

(iv) This can be seen by modifying appropriately the example given in the proof of statement (ii) of this theorem. Indeed, in the aforementioned example, the functions $f_{k}$ have disjoint supports; hence, $\left(\sum_{k}\left|f_{k}\right|^{\alpha}\right)^{1 \alpha}=\sum_{k} f_{k}=$ $\left|\sum_{k} \varepsilon_{k} f_{k}\right|$. Thus, the same argument applies. The second part of the statement is obvious, for a $p$-convex Banach lattice is of Rademacher type $p$. This completes the proof. 
The restriction to the parameter space $T=[0,1]$ and the governing Lebesgue measure is not essential. One can easily extend the results to an arbitrary separable $\sigma$-finite measure space $S$. Also, the extension of main results (Theorems 1 and 2) to strictly stable processes is direct, through the appropriate symmetrization (cf., e.g., [KS3, Proposition 5.1 and p. 774]).

\section{REFERENCES}

[CRW] S. Cambanis, J. Rosiński, and W. A. Woyczyński, Convergence of quadratic forms in p-stable random variables and $\theta_{p}$-randonifying operators, Ann. Probab. 13 (1985), 885-897.

[F] W. Feller, An introduction to probability theory and its applications, 2nd ed., Wiley, New York, 1971.

[Kri] J. Krivine, Theoremes de factorisation dans les espaces reticules, Seminaire Maurey-Schwartz, Exposes 22-23 (1973-74), École Polytechnique, Paris.

[KS1] W. Krakowiak and J. Szulga, Hypercontraction principle and random multilinear forms in Banach spaces, Probab. Theory Related Fields 77 (1988), 325-342.

[KS2] _ Multilinear random forms, Ann. Probab. 14 (1988), 955-973.

[KS3] W. Krakowiak and J. Szulga, A multiple stochastic integral with respect to a strictly p-stable random measure, Ann. Probab. 16 (1988), 764-777.

[KaS] O. Kallenberg and J. Szulga, Multiple integration with respect to Poisson and Lévy processes, Probab. Theory Related Fields 83 (1989), 101-134.

[LT] J. Lindenstrauss and L. Tzafriri, Classical Banach spaces, II. Function spaces, SpringerVerlag, New York, 1979.

[M] J. Musielak, Orlicz spaces and modular spaces, Lecture Notes in Math., vol. 1034, SpringerVerlag, New York, 1983.

[Ru] W. Rudin, Functional analysis, McGraw-Hill, New York, 1973.

[RW] J. Rosiński and W. A. Woyczyński, On Itô stochastic integration with respect to p-stable motion: Inner clock, integrability of sample paths, double and multiple integrals, Ann. Probab. 14 (1986), 271-286.

[S1] J. Szulga, A note on hypercontractivity of $\alpha$-stable random variables, Ann. Probab. 18 (1990), 1746-1758.

[S2] _ Limit theorems of some randomized nonlinear functionals of empirical measures, Auburn University, preprint, 1991.

[S3] _ Limit distributions of $U$-statistics resampled by symmetric stable laws, Probab. Theory Related Fields 94 (1990), 83-90.

Department of Mathematics, Auburn University, Auburn University, Alabama 368495310

E-mail address: szulga@ducvax. auburn.edu

szulga@auducvax.bitnet 